\title{
Asymptotic and non-asymptotic estimates for multivariate Laplace integrals
}

\author{
Maria Rosaria Formica ${ }^{1, *}$, Eugeny Ostrovsky ${ }^{2}$, Leonid Sirota $^{2}$ \\ ${ }^{1}$ Università degli Studi di Napoli Parthenope, Napoli, Italy \\ ${ }^{2}$ Bar-Ilan University, Department of Mathematic and Statistics, Ramat Gan, Israel
}

Abstract We derive bilateral asymptotic as well as non-asymptotic estimates for the multivariate Laplace integrals. Furthermore, we provide multidimensional Tauberian theorems for exponential integrals.

Keywords Laplace or exponential integrals, Fenchel-Morau theorem, random variable and random vector, exponential and ordinary tail of distribution, measurable space, Lebesgue measure, regional and ordinary Young-Fenchel transform, saddle-point method, Cramer's condition, moment generating functions, regular and slowly varying functions.

AMS 2010 subject classifications 44A10, 60B05, 26A12, 40E05.

DOI: $10.19139 /$ soic-2310-5070-806

\section{Definitions. Notations. Previous results. Statement of problem.}

Let $\mathbb{R}^{d}, d=1,2, \ldots$, be the ordinary $d$-dimensional numerical (Euclidean) space

$$
\mathbb{R}^{d}=\{x=\vec{x}=\{x(1), x(2), \ldots, x(d)\}, x(j) \in \mathbb{R}, j=1,2, \ldots, d\},
$$

and let $(X, B, \mu)$ be a non-trivial measurable space equipped with sigma-finite Borelian measure $\mu$, where $X$ is a measurable subset of $\mathbb{R}^{d}$ having measure $\mu(X) \in(0, \infty]$.

Introduce the following subset of the whole space $\mathbb{R}^{d}$

$$
\mathbb{R}^{d}(Z) \stackrel{\text { def }}{=}\left\{x=\vec{x}: \min _{i} x(i) \geq Z\right\}, Z=\mathrm{const} \geq 1 .
$$

We will impose in the sequel the following condition on the set $X$.

\section{Condition 1.1.}

$$
\exists Z_{0}=\text { const } \geq 1: \forall Z \geq Z_{0} \Rightarrow X \cap \mathbb{R}^{d}(Z) \neq \emptyset,
$$

say for all the values $Z \geq 1$ sufficiently large.

Denote

$$
\lambda=\vec{\lambda} \in \mathbb{R}^{d}, \quad(x, \lambda)=x \cdot \lambda=\sum_{i=1}^{d} \lambda(i) x(i), \quad|x|=\sqrt{(x, x)},
$$

\footnotetext{
*Correspondence to: Maria Rosaria Formica (Email: mara.formica@ uniparthenope.it). Università degli Studi di Napoli Parthenope, via Generale Parisi 13, Palazzo Pacanowsky, 80132, Napoli, Italy.
}

ISSN 2310-5070 (online) ISSN 2311-004X (print)

Copyright (C) 2019 International Academic Press 
so that $\operatorname{dim}(x)=\operatorname{dim}(\lambda)=d$.

Define also

$$
\begin{gathered}
\mathbb{R}_{+}^{d}=\{x=\vec{x}=\{x(1), x(2), \ldots, x(d)\}, \quad x(j) \geq 0, j=1,2, \ldots, d\}, \\
\Lambda(\lambda)=\Lambda=\min _{i} \lambda(i), \quad \lambda \in \mathbb{R}_{+}^{d} ;
\end{gathered}
$$

correspondingly

$$
\underline{x}=\Lambda(x)=\min _{i} x(i), \quad x \in X .
$$

Let also $\zeta=\zeta(x), x \in X$, be a measurable numerical valued continuous function $\zeta: X \rightarrow \mathbb{R}$.

We assume, furthermore, that $\mu(X)=\infty$, as long as the opposite (probabilistic) case is trivial for us.

Definition 1. The following integral

$$
I(\lambda)=I[\zeta](\lambda):=\int_{X} e^{(\lambda, x)-\zeta(x)} \mu(d x)
$$

is named Laplace or exponential integral.

In this paper we provide asymptotical as well as non-asymptotical upper and lower estimates of the Laplace integral $I[\zeta](\lambda)=I(\lambda)$, for all sufficiently large values of the real vector parameter $\lambda=\vec{\lambda} \in \mathbb{R}_{+}^{d}, d=1,2,3, \ldots$, for $\Lambda(\lambda) \geq 1$ and when $\Lambda \rightarrow \infty$; we obtain direct estimations of $I(\lambda)$ assuming, of course, its convergence for all the sufficiently large values of the parameter $|\lambda|:=\sqrt{(\lambda, \lambda)}$.

Furthermore we also obtain an inverse evaluation, i.e. we deduce the bilateral bounds for the source function $\zeta=\zeta(x), \underline{x} \geq 1, \Lambda(x) \rightarrow \infty$, through its integral transform $I[\zeta](\lambda)$, with an inverse approach.

The case of other "octants", for instance, $\lambda \in \mathbb{R}_{-}^{d \stackrel{\text { def }}{=}}\{\vec{\lambda}\}, \lambda(j)<0$ and $\Lambda_{-}=\max _{i} \lambda(i) \rightarrow-\infty$, may be investigated quite analogously.

The one-dimensional case $d=1$ was considered in [6, 22, 23]; a preliminary result may be found in [25]. The interested reader can find some other results concerning asymptotic approximations of exponential integrals of the type (2), in one-dimensional case, e.g. in [27, 28]. Integrals of the type (2) have been recently considered also in [21].

We will generalize the main results obtained in the articles [22, 23, 25], where are described also some applications of these estimates, in particular, in the probability theory. Namely, we significantly weaken the restrictions in the mentioned articles and obtain the reverse estimates. The estimates given below may be considered in turn as a generalization of the classical saddle-point method ([11], see also [24]).

The paper is organized as follows. In Section 2 and in Section 3 we deduce respectively an upper and a lower direct estimate for the Laplace integral $I(\lambda)$; Section 4 and Section 5 contain an investigation of the inverse problem and, respectively, an upper and a lower estimate for the source function through the exponential integral. In Section 6 we consider the multidimensional Tauberian theorems for exponential integrals; in Section 7 we describe an example. The last Section contains the concluding remarks.

Most studies concerning multidimensional Tauberian theorems came to the light in the second half of seventies of the twentieth century in connection with many applications in the theory of differential equations, mathematical physics and probability theory (see, for example, [37] and references therein). 
For example, in the probability theory, they play an important role to establish the connection between the behavior of tail of distribution for a random variable and the asymptotic one of its Moment Generation Function (see [3]).

Denote, as usually,

$$
\begin{gathered}
\overrightarrow{0}=\{0,0, \ldots, 0\}, \overrightarrow{1}=\{1,1, \ldots, 1\} ; \operatorname{dim} \overrightarrow{0}=\operatorname{dim} \overrightarrow{1}=d ; \\
\vec{a} \geq \vec{b} \Leftrightarrow a(i) \geq b(i), \quad \forall i=1,2, \ldots, d ; \\
\mathbb{R}_{+}^{d}(1) \stackrel{\text { def }}{=}\{x=\vec{x} \geq \overrightarrow{1}\} .
\end{gathered}
$$

Let us mention briefly a possible application. Recall that the so-called (multivariate) moment generating function (MGF) for the random vector (r.v.) $\vec{\xi}$ is defined by the equality

$$
\begin{aligned}
\exp \left(\phi_{\xi}(\lambda)\right) & \stackrel{\text { def }}{=} \mathbf{E} \exp (\vec{\xi} \cdot \vec{\lambda}) \\
& =\mathbf{E} \exp \left[\sum_{i=1}^{d} \xi(i) \lambda(i)\right]=\int_{\mathbb{R}^{d}} \exp (\vec{\xi}(\omega) \cdot \vec{\lambda}) \mathbf{P}(d \omega) \\
& =\int_{\mathbb{R}^{d}} e^{(\lambda, x)} f_{\xi}(x) d x=\int_{\mathbb{R}^{d}} e^{(\lambda, x)-\ln \left(1 / f_{\xi}(x)\right)} d x
\end{aligned}
$$

where $f_{\xi}(x)$ denotes the density of the r.v. $\xi$, if there exists.

So, the MGF function $\exp \left(\phi_{\xi}(\lambda)\right)$ is, on the other terms, the multivariate Laplace integral.

It will be presumed that the r.v. $\xi$ satisfies the so-called Cramer's condition:

$$
\exists \delta=\text { const }>0: \forall \lambda,|\lambda|<\delta \Rightarrow \phi_{\xi}(\lambda)<\infty
$$

and that the density function there exists.

Recall that the well-known Young-Fenchel or Legendre transform for a function $\zeta: X \rightarrow \mathbb{R}$ is defined as follows

$$
\zeta^{*}(\lambda) \stackrel{\text { def }}{=} \sup _{x \in X}(\lambda \cdot x-\zeta(x)), \quad \lambda \in \mathbb{R}^{d}
$$

If a function $\phi=\phi(\lambda)$ is defined in a set $V$ and is finite, i.e. $\operatorname{dom}[\phi]=\mathrm{V}$, convex or not, one can define formally

$$
\phi(\lambda)=+\infty, \lambda \notin V
$$

hence

$$
\phi^{*}(x) \stackrel{\text { def }}{=} \sup _{\vec{\lambda} \in V}(\vec{\lambda} \cdot \vec{x}-\phi(\vec{\lambda})), \quad x \in \mathbb{R}_{+}^{d}
$$

This notion plays an important role in the probability theory. Namely, let $\xi=\vec{\xi}$ be a random vector for which

$$
\mathbf{E} \exp (\lambda \cdot \xi) \leq \exp (\phi(\lambda)), \quad \lambda \in \mathbb{R}_{+}^{d} .
$$

Then 


$$
T_{\xi}(x) \leq \exp \left(-\phi^{*}(x)\right), \quad x \in \mathbb{R}_{+}^{d},
$$

where $T_{\xi}=T_{\xi}(x)$ denotes the tail function for the r.v. $\xi$ :

$$
T_{\xi}(x) \stackrel{\text { def }}{=} \mathbf{P}(\vec{\xi} \geq \vec{x}), \quad x \in \mathbb{R}_{+}^{d},
$$

the so-called generalized Chernoff's inequality, see e.g. [7, 8, 23].

Moreover, this assertion may be reversed under some natural conditions (smoothness, convexity, etc.) in the following sense. Suppose $d=1$ (one-dimensional case) and that the last estimate (6) holds true. Then, under appropriate conditions (see [23]),

$$
\mathbf{E} \exp (\lambda \cdot \xi) \leq \exp \left(\phi\left(C_{1} \cdot \lambda\right)\right), \quad \lambda \in \mathbb{R}_{+}^{1}
$$

for some finite constant $C_{1}$.

\section{A direct approach. Upper estimate.}

Let us introduce some preliminary notations and conditions. Let $\zeta=\zeta(x), x \in X$, be a measurable numerical valued continuous function $\zeta: X \rightarrow \mathbb{R}$. Put

$$
K(\varepsilon)=K[X, \mu, \zeta](\varepsilon):=\int_{X} e^{-\varepsilon \zeta(x)} \mu(d x),
$$

here and in the sequel $\varepsilon=$ const $\in(0,1)$.

Lemma 1. Assume $X=\mathbb{R}_{+}^{d}$. Let $\mu$ be the classical Lebesgue measure and let $\zeta=\zeta(x), x \in \mathbb{R}_{+}^{d}$, be a nonnegative strictly convex continuous differentiable function. Then the function $K(\varepsilon), \varepsilon>0$, defined by (9), satisfies the following estimate

$$
K(\varepsilon) \leq C[\zeta, d] \exp \left(-C_{0} \varepsilon\right) \varepsilon^{-d}, C_{0} \in \mathbb{R},
$$

where $C$ is a constant depending on $\zeta$ and $d$.

PROOF. There exist positive constants $C_{1}, C_{2}, \ldots, C_{d}$ and a number $C_{0} \in \mathbb{R}$ such that

$$
\zeta(\vec{x}) \geq C_{0}+\sum_{i=1}^{d} C(i) x(i) .
$$

Indeed, one can apply the well-known Fenchel-Morau theorem

$$
\zeta(x)=\sup _{y \in \mathbb{R}_{+}^{d}}\left((x, y)-\zeta^{*}(y)\right)
$$

so that, for an arbitrary $y_{0} \in \mathbb{R}_{+}^{d}$,

$$
\zeta(x) \geq\left(x, y_{0}\right)-\zeta^{*}\left(y_{0}\right)
$$


Therefore

$$
\begin{aligned}
K(\varepsilon) & \leq \int_{\mathbb{R}_{+}^{d}} \exp \left[-\varepsilon\left(C_{0}+\sum_{i=1}^{d} C(i) x(i)\right)\right] \prod_{i=1}^{d} d x(i) \\
& =\left[\prod_{i=1}^{d} C(i)\right]^{-1} e^{-C_{0} \varepsilon} \varepsilon^{-d} .
\end{aligned}
$$

Furthermore, define

$$
Z(\varepsilon)=Z[X, \mu, \zeta](\varepsilon):=\int_{X} \exp (\zeta(x(1-\varepsilon))-\zeta(x)) \mu(d x) .
$$

Definition 2. Let $D \subset X$ be a non-empty subset of the whole set $X$. We define the so-called regional Young-Fenchel transform for the function $\zeta(\cdot)$ by

$$
\zeta^{*}[D](\lambda) \stackrel{\text { def }}{=} \sup _{x \in D}(\lambda \cdot x-\zeta(x)), \lambda \in \mathbb{R}^{d}
$$

so that

$$
\zeta^{*}[X](\lambda)=\zeta^{*}(\lambda)
$$

We present now three methods for an upper estimate of $I(\lambda)$ for sufficiently large values of the real parameter $|\lambda|$.

A. First of all note that if the measure $\mu$ is bounded, i.e. $\mu(X)=M \in(0, \infty)$, then the integral $I(\lambda)$ satisfies a very simple estimate

$$
I(\lambda) \leq M \cdot \sup _{x \in X} \exp (\lambda x-\zeta(x))=M \cdot \exp \left(\zeta^{*}(\lambda)\right) .
$$

Let now $\mu(X)=\infty$ and let $\varepsilon=$ const $\in(0,1)$.

B. It will be presumed the finiteness of the integral $K(\varepsilon)=K[X, \mu, \zeta](\varepsilon)$ at least for some positive value $\varepsilon_{0} \in(0,1)$, i.e.

$$
K(\varepsilon)<\infty \forall \varepsilon \geq \varepsilon_{0} .
$$

In [23] was proved, for the one-dimensional case $d=1$, the following estimate

$$
I(\lambda) \leq K(\varepsilon) \cdot \exp \left\{(1-\varepsilon) \zeta^{*}\left(\frac{\lambda}{1-\varepsilon}\right)\right\} \leq K(\varepsilon) \cdot \exp \left\{\zeta^{*}\left(\frac{\lambda}{1-\varepsilon}\right)\right\} .
$$

The general case may be investigated quite analogously. In detail, let $\varepsilon \in(0,1)$ be some number for which $K(\varepsilon) \in(0, \infty)$. Consider the following probability measure, more precisely, the family of probability measures

or, symbolically,

$$
\nu_{\varepsilon}(A)=\frac{1}{K(\varepsilon)} \int_{A} e^{-\varepsilon \zeta(x)} \mu(d x)
$$

$$
\nu_{\varepsilon}(d x)=\frac{1}{K(\varepsilon)} e^{-\varepsilon \zeta(x)} \mu(d x)
$$

so that

$$
\nu_{\varepsilon}(X)=\int_{X} \nu_{\varepsilon}(d x)=1
$$


We have

$$
\begin{aligned}
\frac{I(\lambda)}{K(\varepsilon)} & =\int_{X} e^{(\lambda, x)-(1-\varepsilon) \zeta(x)} \nu_{\varepsilon}(d x) \\
& \leq \exp \left\{\sup _{x \in X}[(\lambda, x)-(1-\varepsilon) \zeta(x)]\right\} \\
& =\exp \left\{(1-\varepsilon) \zeta^{*}(\lambda /(1-\varepsilon))\right\}
\end{aligned}
$$

So, the relation (12) is proved.

As a slight consequence we have

$$
\begin{gathered}
I(\lambda) \leq \inf _{\varepsilon \in(0,1)}\left[K(\varepsilon) \cdot \exp \left\{(1-\varepsilon) \zeta^{*}\left(\frac{\lambda}{1-\varepsilon}\right)\right\}\right] \\
I(\lambda) \leq \inf _{\varepsilon \in(0,1)}\left[K(\varepsilon) \cdot \exp \left\{\zeta^{*}\left(\frac{\lambda}{1-\varepsilon}\right)\right\}\right] .
\end{gathered}
$$

C. We present here an opposite method, which was introduced in a particular case in [22], [23]. Define the following integral

$$
Z(\varepsilon)=Z[\zeta, \mu, X](\varepsilon):=\int_{X} e^{\zeta((1-\varepsilon) x)-\zeta(x)} \mu(d x),
$$

if, of course, it is finite at least for some value $\varepsilon \in(0,1)$.

Applying the well-known Young inequality

$$
(\lambda, x) \leq \zeta((1-\varepsilon) x)+\zeta^{*}(\lambda /(1-\varepsilon)),
$$

we have

$$
I(\lambda) \leq e^{\zeta^{*}(\lambda /(1-\varepsilon))} \int_{X} e^{\zeta((1-\varepsilon) x)-\zeta(x)} \mu(d x)=Z(\varepsilon) e^{\zeta^{*}(\lambda /(1-\varepsilon))}
$$

Of course

$$
I(\lambda) \leq \inf _{\varepsilon \in(0,1)}\left[Z(\varepsilon) e^{\zeta^{*}(\lambda /(1-\varepsilon))}\right]
$$

D. Denote

$$
R(\varepsilon)=R[X, \mu, \zeta](\varepsilon):=\min (K(\varepsilon), Z(\varepsilon)), \quad \varepsilon \in(0,1),
$$

where $K(\varepsilon)$ and $Z(\varepsilon)$ are defined respectively in (9) and (15).

We conclude

$$
I(\lambda) \leq R[X, \mu, \zeta](\varepsilon) e^{\zeta^{*}(\lambda /(1-\varepsilon))}
$$

Furthermore, we will use the following elementary inequality

$$
1+\varepsilon<\frac{1}{1-\varepsilon} \leq 1+2 \varepsilon, \quad 0<\varepsilon \leq 1 / 2 .
$$

Now we put $\phi(\lambda):=\zeta^{*}(\lambda)$ and we define 


$$
\begin{gathered}
\pi_{\kappa}(\lambda) \stackrel{\text { def }}{=} \frac{\kappa}{\left(\lambda \cdot \zeta^{*^{\prime}}(\lambda)\right)}=\frac{\kappa}{\left(\lambda \cdot \phi^{\prime}(\lambda)\right)}, \quad \kappa=\text { const } \in(0, \infty), \\
\pi(\lambda)=\pi_{1}(\lambda) \stackrel{\text { def }}{=} \frac{1}{\left(\lambda \cdot \zeta^{*^{\prime}}(\lambda)\right)}=\frac{1}{\left(\lambda \cdot \phi^{\prime}(\lambda)\right)},
\end{gathered}
$$

alike the ones considered in [29, chapter 3], and suppose that

$$
\lim _{\Lambda(\lambda) \rightarrow \infty} \pi(\lambda)=0
$$

so that the value $\Lambda_{0}=\Lambda(\kappa)$ may be chosen such that

$$
\forall \lambda: \min _{i} \lambda(i) \geq \Lambda(\kappa) \Rightarrow \pi_{\kappa}(\lambda) \leq 1 / 2 .
$$

Let us impose the following condition on the function $\phi(\cdot)$ :

$$
C(\phi)=C(\phi, \kappa):=\sup _{\min _{i} \lambda(i) \geq \Lambda(\kappa)}\left[\phi\left(\lambda+\frac{2 \lambda}{\left(\lambda, \phi^{\prime}(\lambda)\right)}\right)-\phi(\lambda)\right]<\infty .
$$

Define also

$$
r(\lambda)=r[\zeta, \kappa](\lambda) \stackrel{\text { def }}{=} R[X, \mu, \zeta]\left(\pi_{\kappa}(\lambda)\right),
$$

where $R$ is defined in (17) and $\pi_{\kappa}(\lambda)$ in (19).

Choosing $\varepsilon=\pi(\lambda)=\pi_{1}(\lambda)$ with $\lambda$ such that $\min _{i} \lambda(i) \geq \Lambda(1)$, we have the following

Theorem 1. Let $\zeta=\zeta(x), x \in X$, be a measurable numerical valued continuous function $\zeta: X \rightarrow \mathbb{R}$. If the function $\phi(\cdot)=\zeta^{*}(\cdot)$ satisfies the condition (22), then

$$
I(\lambda) \leq e^{C(\phi)} r(\lambda) e^{\zeta^{*}(\lambda)}
$$

where $r(\lambda)$ is defined by (23).

Example 1. Assume in addition $R(\varepsilon) \leq C_{1} \varepsilon^{-\beta}, \beta=$ const $\in(0, \infty), \varepsilon \in(0,1)$; then

$$
I(\lambda) \leq C_{1} e^{C(\phi)} \varepsilon^{-\beta} e^{2 \varepsilon / \pi(\lambda)} e^{\phi(\lambda)}, \lambda(i) \geq \Lambda,
$$

and, after the minimization over $\varepsilon$,

$$
I(\lambda) \leq C_{1} e^{C(\phi)} 2^{\beta} \beta^{-\beta} e^{\beta} \pi_{1}^{-\beta}(\lambda) e^{\zeta^{*}(\lambda)} .
$$

E. Let us consider an arbitrary simple partition $X=X_{0} \cup X_{1}, X_{0} \cap X_{1}=\emptyset$ of the whole set $X$ onto two disjoint measurable subsets. We split the integral $I(\lambda)$ into two ones

$$
I(\lambda)=\int_{X_{0}} \exp (\lambda x-\zeta(x)) \mu(d x)+\int_{X_{1}} \exp (\lambda x-\zeta(x)) \mu(d x)=I_{0}+I_{1},
$$

and we apply the foregoing estimates, so that

$$
I_{0} \leq \mu\left(X_{0}\right) \exp \left[\sup _{x \in X_{0}}(\lambda x-\zeta(x))\right]=\mu\left(X_{0}\right) \exp \left[\zeta^{*}\left[X_{0}\right](\lambda)\right],
$$




$$
\begin{aligned}
I_{1} & \leq R\left[X_{1}, \mu, \zeta\right](\varepsilon) \exp \left[\sup _{x \in X_{1}}(\lambda x /(1-\varepsilon)-\zeta(x))\right] \\
& =R\left[X_{1}, \mu, \zeta\right](\varepsilon) \exp \left[\zeta^{*}\left[X_{1}\right](\lambda /(1-\varepsilon)] .\right.
\end{aligned}
$$

We obtain the following compound estimate.

Lemma 2. Let

$$
\begin{gathered}
W[X, \mu, \zeta, \varepsilon](\lambda)=: \mu\left(X_{0}\right) \exp \left[\zeta^{*}\left[X_{0}\right](\lambda)\right]+R\left[X_{1}, \mu, \zeta\right](\varepsilon) \exp \left[\zeta^{*}\left[X_{1}\right](\lambda /(1-\varepsilon)],\right. \\
W_{0}[X, \mu, \zeta](\lambda)=\inf _{\varepsilon \in(0,1)} W[X, \mu, \zeta, \varepsilon](\lambda) .
\end{gathered}
$$

If there exists a positive constant $c$ such that

$$
\forall \lambda,|\lambda| \geq c \Rightarrow W_{0}[X, \mu, \zeta](\lambda)<\infty
$$

then, $\forall \lambda:|\lambda| \geq c$ and $\forall \varepsilon \in(0,1)$,

$$
I(\lambda) \leq W[X, \mu, \zeta, \varepsilon](\lambda)
$$

As a slight consequence we have

$$
I(\lambda) \leq W_{0}[X, \mu, \zeta](\lambda), \quad|\lambda| \geq c
$$

Remark 1. Introduce the following condition on the function $\zeta(\cdot)$ :

$$
\exists C_{1} \in[1, \infty): W_{0}[X, \mu, \zeta](\lambda) \leq \exp \left\{\zeta^{*}\left(C_{1} \lambda\right)\right\},|\lambda| \geq c .
$$

This condition is satisfied if, for example, the function $\zeta=\zeta(x), x \in X$, is regular varying:

$$
\zeta(\lambda)=|\lambda|^{m} L(|\lambda|), \quad|\lambda| \geq 1,
$$

where $m=$ const $>0,|\cdot|$ is the ordinary Euclidean norm (or an other arbitrary non-degenerate vector one) and $L=L(r), r \geq 1$, is some positive continuous slowly varying function as $r \rightarrow \infty$, and we suppose

$$
\forall A \in B \Rightarrow \mu(A)=\int_{A}|x|^{\alpha} M(|x|) d x, \quad \alpha=\text { const }>-d,
$$

where, as before, $M=M(r), r \geq 1$, is some positive continuous slowly varying function as $r \rightarrow \infty$. Briefly, $\mu(d x)=|x|^{\alpha} M(|x|) d x$. For the integral $K(\varepsilon)$ defined in (9) we have the inequality

$$
K(\varepsilon) \leq \bar{K}(\varepsilon), \quad \varepsilon \in(0,1),
$$

where

$$
\bar{K}(\varepsilon):=\int_{\mathbb{R}^{d}} \exp \left(-\varepsilon|x|^{m} L(|x|)\right)|x|^{\alpha} M(|x|) d x .
$$

One can apply the spherical coordinates:

$$
\bar{K}(\varepsilon)=\frac{\pi^{d / 2}}{\Gamma(d / 2+1)} K_{0}(\varepsilon),
$$


where

$$
K_{0}(\varepsilon)=\int_{0}^{\infty} \exp \left(-\varepsilon r^{m} L(r)\right) r^{\alpha+d-1} M(r) d r .
$$

We obtain, after the substitution $r^{m} \varepsilon=y, d r=m^{-1} y^{1 / m-1} \varepsilon^{-1 / m} d y$,

$$
\begin{aligned}
Z_{m}(\varepsilon) & \stackrel{\text { def }}{=} m \varepsilon^{(\alpha+d) / m} K_{0}(\varepsilon) \\
& =\int_{0}^{\infty} y^{(\alpha+d) / m-1} \exp \left(-y L\left(y^{1 / m} \varepsilon^{-1 / m}\right)\right) M\left(y^{1 / m} \varepsilon^{-1 / m}\right) d y
\end{aligned}
$$

and, as $\varepsilon \rightarrow 0+$,

$$
\begin{aligned}
Z_{m}(\varepsilon) & \sim M\left(\varepsilon^{-1 / m}\right) \int_{0}^{\infty} e^{-y L\left(\varepsilon^{-1 / m}\right)} y^{(\alpha+d) / m-1} d y \\
& =M\left(\varepsilon^{-1 / m}\right) \Gamma((\alpha+d) / m) L^{-(\alpha+d) / m}\left(\varepsilon^{-1 / m}\right),
\end{aligned}
$$

where $\Gamma$ is the classical Gamma function.

To summarize: as $\varepsilon \rightarrow 0+$

$$
m \bar{K}(\varepsilon) \sim \frac{\pi^{d / 2}}{\Gamma(d / 2+1)} \varepsilon^{-(\alpha+d) / m} \Gamma((\alpha+d) / m) \frac{M\left(\varepsilon^{-1 / m}\right)}{L^{(\alpha+d) / m}\left(\varepsilon^{-1 / m}\right)} .
$$

Thus, in this case, the values $K=K(\varepsilon)$ and $R=R(\varepsilon), \varepsilon \in(0,1)$, are finite with concrete estimate following from (32):

$$
m Z(\varepsilon) \leq C[\zeta, m, d] \frac{\pi^{d / 2}}{\Gamma(d / 2+1)} \varepsilon^{-(\alpha+d) / m} \Gamma((\alpha+d) / m) \frac{M\left(\varepsilon^{-1 / m}\right)}{L^{(\alpha+d) / m}\left(\varepsilon^{-1 / m}\right)} .
$$

If the condition of Remark 2.1 is satisfied, then

$$
I(\lambda) \leq \exp \left(\zeta^{*}\left(C_{2} \lambda\right)\right), \quad L(\lambda) \geq 1 .
$$

Theorem 2. Let $X=\mathbb{R}_{+}^{d}$ and $\mu$ be the ordinary Lebesgue measure. Suppose that the random vector $\vec{\xi}$, with non-negative entries $\{\xi(i)\}, i=1,2, \ldots, d$, satisfies the Cramer's condition:

$$
\exists \lambda_{0}=\overrightarrow{\lambda_{0}}=\left\{\lambda_{0}(i)\right\}, i=1,2, \ldots, \lambda_{0}(i)>0: \mathbf{E} \exp \left(\lambda_{0} \cdot \xi\right)<\infty .
$$

Then the integral $K(\varepsilon)$ defined in (9) satisfies the following condition:

$$
\exists \varepsilon_{0}>0: \forall \varepsilon>\varepsilon_{0} \Rightarrow K(\varepsilon)=K[X, \mu, \zeta](\varepsilon)<\infty .
$$

PROOF. Denote, for brevity, $G(x)=G[\xi](x)$, so that

$$
T_{\xi}(\vec{x})=e^{-G(\vec{x})}, x \geq 0 .
$$

It is sufficient to consider only the two-dimensional case: assume

$$
B=B(\lambda, \mu):=\int_{0}^{\infty} \int_{0}^{\infty} e^{\lambda x+\mu y-G(x, y)} d x d y<\infty
$$


for some positive values $\lambda, \mu$. We have

$$
\begin{gathered}
B=\sum_{n=0}^{\infty} \sum_{m=0}^{\infty} \int_{n}^{n+1} \int_{m}^{m+1} e^{\lambda x+\mu y-G(x, y)} d x d y \geq \\
\sum_{n=0}^{\infty} \sum_{m=0}^{\infty} \int_{n}^{n+1} \int_{m}^{m+1} e^{\lambda n+\mu m-G(n+1, m+1)} d x d y=\sum_{n=0}^{\infty} \sum_{m=0}^{\infty} e^{\lambda n+\mu m-G(n+1, m+1)},
\end{gathered}
$$

therefore

$$
\sum_{n=0}^{\infty} \sum_{m=0}^{\infty} e^{\lambda n+\mu m-G(n+1, m+1)}<B(\lambda, \mu)<\infty
$$

so

$$
e^{\lambda n+\mu m-G(n+1, m+1)} \leq B(\lambda, \mu)<\infty, G(n+1, m+1) \leq B e^{-\lambda n-\mu m},
$$

and finally

$$
(\lambda \mu)^{-1} K(\varepsilon)<\int_{0}^{\infty} \int_{0}^{\infty} e^{-\varepsilon G(x, y)} d x d y \leq \sum_{n=0}^{\infty} \sum_{m=0}^{\infty} \exp \{-\varepsilon[\lambda(n-1)+\mu(m-1)]\}<\infty,
$$

if

$$
\varepsilon>\varepsilon_{0}:=\max \left(\lambda^{-1}, \mu^{-1}\right)
$$

as desired.

\section{A direct approach. Lower estimate.}

We introduce the following additional notations. Define

$$
\begin{gathered}
S(\lambda, x)=(\lambda, x)-\zeta(x), \\
\operatorname{argmax}_{x \in X} S(\lambda, x)=\left\{x \in X: S(\lambda, x)=\zeta^{*}(\lambda)\right\} .
\end{gathered}
$$

Consider $x_{0}=x_{0}(\lambda) \in \operatorname{argmax}_{x \in X}(\lambda, x)$; obviously, the value $x_{0}=x_{0}(\lambda)$ may be non-unique.

Furthermore, we introduce the variables

$$
\begin{gathered}
X_{0}=X_{0}(\varepsilon)=X_{0}(\varepsilon, \lambda):=\left\{x \in X: S(\lambda, x) \geq \zeta^{*}(\lambda(1-\varepsilon))\right\}, \quad \varepsilon \in(0,1), \\
U(\varepsilon)=U[\zeta](\varepsilon, \lambda):=\int_{X_{0}(\varepsilon)} \mu(d x)=\mu\left(X_{0}(\varepsilon, \lambda)\right) .
\end{gathered}
$$

Theorem 3. Let $U$ be defined in (35) and let $\varepsilon \in(0,1)$ be such that $U(\varepsilon)>0$. Then, for sufficiently large values $\min _{i} \lambda(i) \geq \Lambda=$ const $\geq 1$, we have

$$
I(\lambda) \geq U[\zeta](\varepsilon, \lambda) \exp \left(\zeta^{*}(\lambda(1-\varepsilon))\right), \varepsilon \in(0,1), \min _{i} \lambda(i) \geq \Lambda .
$$


Of course,

$$
I(\lambda) \geq \sup _{\varepsilon \in(0,1)}\left[U[\zeta](\varepsilon, \lambda) \exp \left(\zeta^{*}(\lambda(1-\varepsilon))\right)\right], \min _{i} \lambda(i) \geq \Lambda
$$

PROOF.

$$
\begin{aligned}
I(\lambda) & =\int_{X} \exp [\lambda x-\zeta(x)] \mu(d x) \geq \int_{X_{0}} \exp [\lambda x-\zeta(x)] \mu(d x) \\
& \geq \int_{X_{0}} \exp \left[\zeta^{*}(\lambda(1-\varepsilon))\right] \mu(d x)=U[\zeta](\varepsilon, \lambda) \exp \left[\zeta^{*}(\lambda(1-\varepsilon))\right] .
\end{aligned}
$$

As a slight consequence we get:

\section{Corollary 1.}

$$
I(\lambda) \geq U[\zeta](\varepsilon, \lambda) \exp \left(\zeta^{*}(\lambda)-\varepsilon\left(\lambda, \zeta^{*^{\prime}}(\lambda)\right)\right),
$$

and, if we choose $\varepsilon=\pi_{\kappa}(\lambda)$ defined in (19),

$$
I(\lambda) \geq U[\zeta]\left(\pi_{\kappa}(\lambda), \lambda\right) \exp \left(\zeta^{*}(\lambda)-\kappa\right) .
$$

Let us define the following function

$$
V(\lambda)=V[\zeta](\lambda) \stackrel{\text { def }}{=} \sup _{\kappa>0}\left\{U[\zeta]\left(\pi_{\kappa}(\lambda), \lambda\right) e^{-\kappa}\right\}
$$

so, by (36), we have

$$
I(\lambda) \geq V[\zeta](\lambda) e^{\zeta^{*}(\lambda)}, \min _{i} \lambda(i) \geq \Lambda .
$$

For instance, it is reasonable to suppose in addition, see e.g. Example 3.1 below, that

$$
U[\zeta]\left(\pi_{\kappa}(\lambda), \lambda\right) \geq \gamma \Lambda^{\alpha} \kappa^{\beta}, \alpha, \beta, \gamma=\text { const } \in(0, \infty)
$$

then

$$
I(\lambda) \geq \gamma(\beta / e)^{\beta} \Lambda^{\alpha} e^{\zeta^{*}(\lambda)}, \Lambda=\min _{i} \lambda(i) \geq e .
$$

Let us consider the following example.

Example 2. Suppose that $X=\mathbb{R}_{+}^{d}, d \mu=d x$ and that the function $\zeta=\zeta(x), x \in X=\mathbb{R}_{+}^{d}$ is non-negative, strictly convex, twice continuous and differentiable as well as its conjugate $\zeta^{*}(\lambda)$ and such that its second (matrix) derivative

$$
\zeta^{\prime \prime}(x)=\left\{\frac{\partial^{2} \zeta(x)}{\partial x(i) \partial x(j)}\right\}, i, j=1,2, \ldots, d
$$

is a strictly positive definite matrix for all sufficiently large values $\min _{i} x(i)$.

Denote also

$$
\zeta^{\prime}(x)=\operatorname{grad} \zeta(x)=\left\{\frac{\partial \zeta}{\partial x(i)}\right\}
$$




$$
\begin{gathered}
x_{0}=\vec{x}_{0}[\zeta](\lambda)=x_{0}[\zeta](\lambda)=\operatorname{argmax}_{x \in R_{+}^{d}} S(\lambda, x)=\operatorname{argmax}_{x \in R_{+}^{d}}[(\lambda, x)=(\lambda, x)-\zeta(x)], \\
\Delta=\Delta(\lambda, x)=S(\lambda, x)-S\left(\lambda(1-\varepsilon), x_{0}(\lambda)\right),
\end{gathered}
$$

so that

$$
\operatorname{grad} \zeta\left(x_{0}\right)=\lambda, \quad \lim _{\Lambda \rightarrow \infty} x_{0}[\zeta](\lambda)=\infty
$$

and

$$
\begin{aligned}
X_{0}(\varepsilon, \lambda) & =\left\{x \in R_{+}^{d}: S(\lambda, x) \geq \zeta^{*}(\lambda(1-\varepsilon))\right. \\
& =\left\{x \in R_{+}^{d}: S(\lambda, x) \geq S\left(\lambda(1-\varepsilon), x_{0}(\lambda)\right)\right\} .
\end{aligned}
$$

We deduce after simple calculations, using Taylor's formula, that the set $X_{0}(\varepsilon, \lambda)$ is asymptotical equivalent, as $\varepsilon \rightarrow 0+$, to the following one (multidimensional ellipsoid)

$$
\tilde{X}_{0}=\left\{x:\left(\zeta^{\prime \prime}\left(x_{0}\right)\left(x-x_{0}\right),\left(x-x_{0}\right)\right) \leq \varepsilon\left(\lambda, x_{0}\right)\right\},
$$

in the sense that

$$
\lim _{\varepsilon \rightarrow 0+} \frac{\mu\left(\tilde{X}_{0}\right)}{\mu\left(X_{0}\right)}=1
$$

The case when the value $\varepsilon=\varepsilon(\lambda)$ is dependent on $\lambda$, but such that

$$
\lim _{\Lambda \rightarrow \infty} \varepsilon(\lambda)=0,
$$

can not be excluded.

It is no hard to calculate the "volume" of the ellipsoid $\tilde{X}_{0}$ :

$$
\mu\left(\tilde{X}_{0}\right)=\frac{\pi^{d / 2}\left[2 \varepsilon \cdot\left(\lambda, x_{0}\right)\right]^{d}}{\Gamma(d / 2+1)} \cdot\left\{\operatorname{det} \zeta^{\prime \prime}\left(x_{0}\right)\right\}^{-1 / 2} .
$$

Following

$$
\mu\left(X_{0}\right) \geq C_{0}(d)\left[\varepsilon \cdot\left(\lambda, x_{0}[\zeta](\lambda)\right]^{d} \cdot\left\{\operatorname{det} \zeta^{\prime \prime}\left(x_{0}\right)\right\}^{-1 / 2}\right.
$$

If, for instance, $d=1, m=$ const $>1$,

$$
\zeta(x)=\zeta_{m}(x) \stackrel{\text { def }}{=} x^{m} / m,
$$

then

$$
x_{0}=\lambda^{1 /(m-1)}, \quad \lambda, x \geq 1 ; \quad \mu\left(X_{0}\right) \geq C_{m} \varepsilon^{1 / 2} \lambda^{1 /(m-1)},
$$

and we find, after some calculations,

$$
\begin{aligned}
I_{m}(\lambda) & \stackrel{\text { def }}{=} \int_{0}^{\infty} \exp \left(\lambda x-x^{m} / m\right) d x \\
& \geq\left(\frac{2.5}{m-1}\right)^{1 / 2} \lambda^{(2-m) /(2 m-2)} \exp \left(\lambda^{m^{\prime}} / m^{\prime}\right),
\end{aligned}
$$


where $m^{\prime}=m /(m-1), \lambda \geq \lambda_{0}(m)$ and

$$
\lambda_{0}(m)= \begin{cases}2 & \text { if } 1<m \leq 2 \\ 2\left(\frac{m-2}{2 m-2}\right)^{(m-1) / m} & \text { if } m>2\end{cases}
$$

The last estimate is in full accordance, up to a multiplicative constant, with the exact asymptotic estimates for $I_{m}(\lambda)$, as $\lambda \rightarrow \infty$, which may be found, e.g., in [11, sections 1,2]:

$$
I_{m}(\lambda) \sim \sqrt{2 \pi /(m-1)} \lambda^{(2-m) /(2 m-2)} \exp \left(\lambda^{m^{\prime}} / m^{\prime}\right) .
$$

The upper estimate corresponding to the lower one obtained above, for the integral $I_{m}(\lambda)$, has the form

$$
I_{m}(\lambda) \leq m^{2 / m-1} e^{1 / m} \Gamma(1 / m) \lambda^{1 /(m-1)} \exp \left(\lambda^{m^{\prime}} / m^{\prime}\right), \lambda \geq \lambda_{0}(m) .
$$

\section{Inverse approach. Upper estimation.}

Suppose that there exists a non-negative continuous function $J=J(\lambda)$ such that the integral defined in (2) satisfies

$$
\int_{X} e^{(\lambda, x)-\zeta(x)} \mu(d x) \geq J(\lambda), \quad \lambda \in \mathbb{R}_{+}^{d}
$$

Here we derive the upper bound for the source function $\zeta=\zeta(x)$ for all the sufficiently large values $\Lambda(x)=$ $\min _{i} x(i), i=1,2, \ldots, d$, under appropriate conditions.

Theorem 4. Let $\zeta: X \rightarrow \mathbb{R}$ be a measurable, non-negative, continuous and convex function and let $J=J(\lambda)$ be a non-negative continuous function, satisfying the condition (40). Assume that there exists a constant $C_{12}=$ $C_{12}[\zeta] \in(0, \infty)$ such that

$$
e^{C\left(\zeta^{*}\right)} r(\lambda) e^{\zeta^{*}(\lambda)} \leq e^{\zeta^{*}\left(C_{12}[\zeta](\lambda)\right)}, \lambda \geq \Lambda=\text { const } \geq 1,
$$

where $C\left(\zeta^{*}\right)$ is defined in (22) with $\phi(\cdot)=\zeta^{*}(\cdot)$ and $r(\lambda)$ is defined in (23).

Then

$$
\zeta(x) \leq[\ln J(\cdot)]^{*}\left(C_{12} x\right), \quad \Lambda(x)=\min _{i} x(i) \geq 1 .
$$

PROOF. By (41) and Theorem 1 we have

$$
e^{\zeta^{*}\left(C_{12}[\zeta](\lambda)\right)} \geq J(\lambda), \quad \lambda \geq \Lambda
$$

which implies

$$
\ln J(\lambda) \leq \zeta^{*}\left(C_{12} \lambda\right)
$$

therefore

$$
[\ln J(\cdot)]^{*}(x) \geq \zeta^{* *}\left(x / C_{12}\right) .
$$

By virtue of Fenchel-Moreau Theorem, we get (42). 


\section{Inverse approach. Lower estimation.}

Suppose that there exists a non-negative continuous function $K(\lambda)$ such that the integral defined in (2) satisfies

$$
\int_{X} e^{(\lambda, x)-\zeta(x)} \mu(d x) \leq K(\lambda), \quad \lambda \in \mathbb{R}_{+}^{d}
$$

Here we derive the lower bound for the source function $\zeta=\zeta(x)$.

Theorem 5. Let $\zeta: X \rightarrow \mathbb{R}$ be a measurable, non-negative, continuous and convex function and let $K=K(\lambda)$ be a non-negative continuous function, satisfying the condition (43). Assume that there exists a constant $C_{13}=$ $C_{13}[\zeta] \in(0,1)$ such that

$$
e^{C\left(\zeta^{*}\right)} V[\zeta](\lambda) e^{\zeta^{*}(\lambda)} \geq e^{\zeta^{*}\left(C_{13} \lambda\right)}, \quad \lambda \geq \Lambda=\text { const } \geq 1,
$$

where $C\left(\zeta^{*}\right)$ is defined in (22) with $\phi(\cdot)=\zeta^{*}(\cdot)$ and $V[\zeta](\lambda)$ is defined in (37).

Then

$$
\zeta(x) \geq[\ln K(\cdot)]^{*}\left(C_{13} x\right), \quad \Lambda(x) \geq 1 .
$$

PROOF. By Theorem 3 and its consequences, in particular inequality (38), we have

$$
e^{\zeta^{*}\left(C_{13} \lambda\right)} \leq K(\lambda), \quad \lambda \geq \Lambda
$$

which implies

$$
\ln K(\lambda) \geq \zeta^{*}\left(C_{13} \lambda\right)
$$

Therefore, by virtue of Fenchel-Moreau Theorem, we have (45).

\section{Multivariate Tauberian theorems.}

Preface. Tauberian theorems are named the relations between asymptotical or not-asymptotical behavior of some function (sequence) and correspondent behavior of its certain integral transform, for example, Laplace, Fourier or power series transform, see $[36,19]$. They play a very important role, for example, in the probability theory (see [3]), to establish the connection between the behavior of tail of distribution for a random variable and the asymptotic one of its Moment Generation Function (MGF).

There are many results in this direction for one-dimensional case, as well as asymptotical ones, see e.g. in $[1,4,9,10,12,13,16,17,20,26,33,37]$.

In this Section we investigate multivariate Tauberian theorems describing relations between the function $\zeta=\zeta(x), x \in X$, and its Laplace integral transform $I[\zeta](\lambda), \lambda \in \mathbb{R}^{d}$, when $\Lambda(x) \rightarrow \infty$ or, correspondingly, $\Lambda(\lambda) \rightarrow \infty$.

\section{DIRECT APPROACH.}

Theorem 6. (Upper limit). Under the same assumptions of Theorem 1 for the function $\phi(\lambda)=\zeta^{*}(\lambda)$, if in addition suppose that

$$
\lim _{\min _{i} \lambda(i) \rightarrow \infty} \phi(\lambda)=\infty
$$

and

$$
\lim _{\min _{i} \lambda(i) \rightarrow \infty} \frac{|\ln r(\lambda)|}{\phi(\lambda)}=0,
$$

then

$$
\varlimsup_{\min _{i} \lambda(i) \rightarrow \infty} \frac{\ln I(\lambda)}{\phi(\lambda)} \leq 1 .
$$


PROOF. Choosing $\varepsilon=\varepsilon(\lambda)=\pi(\lambda)=\pi_{1}(\lambda)$ we have, for sufficiently large values $\Lambda(\lambda)=\min _{i} \lambda(i)$,

$$
\frac{\ln I(\lambda)}{\phi(\lambda)} \leq \frac{|\ln r(\lambda)|}{\phi(\lambda)}+\frac{\phi(\lambda+\lambda \pi(\lambda))}{\phi(\lambda)} .
$$

The term on the left hand side tends to zero as $\Lambda \rightarrow \infty$, the limit of the quantity on the right hand side is equal to one. In detail,

$$
\frac{\phi(\lambda+\lambda \pi(\lambda))}{\phi(\lambda)} \geq \frac{\phi(\lambda)}{\phi(\lambda)}=1
$$

On the other hand, from the condition (22) it follows

$$
\phi(\lambda+2 \lambda \pi(\lambda)) \leq C(\phi)+\phi(\lambda)
$$

therefore, by virtue of condition (46),

$$
\frac{\phi(\lambda+\lambda \pi(\lambda))}{\phi(\lambda)} \leq 1+\frac{C_{1}(\phi)}{\phi(\lambda)} \rightarrow 1
$$

as $\Lambda(\lambda) \rightarrow \infty$.

This completes the proof.

Theorem 7. (Lower limit). Under the same assumptions of Theorem 3 for the function $\phi(\lambda)=\zeta^{*}(\lambda)$, if in addition suppose that

$$
\lim _{\Lambda(\lambda) \rightarrow \infty} \frac{\ln V(\lambda)}{\zeta^{*}(\lambda)}=0
$$

where $V$ is defined in (37), then

$$
\lim _{\Lambda(\lambda) \rightarrow \infty} \frac{\ln I(\lambda)}{\zeta^{*}(\lambda)} \geq 1
$$

PROOF. The proof is completely alike to the one based on Theorem 6 and may be omitted.

As consequence of Theorems 6 and 7 we have

Theorem 8. Suppose that all the conditions of Theorems 6 and 7 are satisfied. Then there exists the following limit and

$$
\lim _{\Lambda(\lambda) \rightarrow \infty} \frac{\ln I(\lambda)}{\zeta^{*}(\lambda)}=1
$$

\section{INVERSE APPROACH.}

Theorem 9. (Lower estimate) Assume that the function $\zeta=\zeta(x)$ in the integral (2) is convex and continuous. Suppose that there exists a continuous non-negative function $Q=Q(\lambda), \lambda \in \mathbb{R}^{d}$, for which

$$
\begin{gathered}
\lim _{\Lambda(\lambda) \rightarrow \infty} \frac{\ln V(\lambda)}{Q(\lambda)}=0, \\
\varlimsup_{\Lambda(\lambda) \rightarrow \infty} \frac{\ln I(\lambda)}{Q(\lambda)} \leq 1,
\end{gathered}
$$


and

$$
\lim _{\Lambda(\lambda) \rightarrow \infty} Q(\lambda)=\infty
$$

Then

$$
\varliminf_{\Lambda(x) \rightarrow \infty} \frac{\zeta(x)}{Q^{*}(x)} \geq 1
$$

PROOF. Let $\delta \in(0,1)$ be an arbitrary "small"number. There exists a value $\Lambda_{0}=\Lambda_{0}(\delta)>1$ such that, for all the values $\lambda$,

$$
\Lambda(\lambda) \geq \Lambda_{0} \Rightarrow \ln I(\lambda) \leq(1+\delta) Q(\lambda)
$$

which implies

$$
I(\lambda) \leq \exp ((1+\delta) Q(\lambda))
$$

Now we use the estimate (38) in Corollary 36, so that

$$
V[\zeta](\lambda) e^{\zeta^{*}(\lambda)} \leq \exp ((1+\delta) Q(\lambda))
$$

and

$$
\frac{\zeta^{*}(\lambda)}{Q(\lambda)} \leq \frac{\ln V(\lambda)}{Q(\lambda)}+(1+\delta) \leq(1+2 \delta), \quad \Lambda(\lambda) \geq 2 \Lambda_{0}
$$

Therefore

$$
\zeta^{*}(\lambda) \leq(1+2 \delta) Q(\lambda)
$$

and

$$
\zeta^{* *}(x) \geq \frac{1}{1+2 \delta} Q^{*}\left(\frac{x}{1+2 \delta}\right) .
$$

Applying the Fenchel-Morau Theorem, we conclude the proof.

Theorem 10. (Upper estimate) Assume that the function $\zeta=\zeta(x)$ in the integral (2) is convex and continuous. Suppose that there exists a continuous non-negative function $Q=Q(\lambda), \lambda \in \mathbb{R}^{d}$, for which

$$
\lim _{\Lambda(\lambda) \rightarrow \infty} \frac{\ln r(\lambda)}{Q(\lambda)}=0
$$

where $r(\lambda)$ is defined in (23), and

$$
\begin{gathered}
\varlimsup_{\Lambda(\lambda) \rightarrow \infty} \frac{\ln I(\lambda)}{Q(\lambda)} \geq 1, \\
\lim _{\Lambda(\lambda) \rightarrow \infty} Q(\lambda)=\infty .
\end{gathered}
$$

Then

$$
\varlimsup_{\Lambda(x) \rightarrow \infty} \frac{\zeta(x)}{Q^{*}(x)} \leq 1
$$

PROOF. The proof is quite alike as the one in Theorem 9. Let $\delta \in(0,1)$ be an arbitrary "small"number. There exists a value $\Lambda_{0}=\Lambda_{0}(\delta)>1$ such that, for all the values $\lambda$,

$$
\begin{gathered}
\Lambda(\lambda) \geq \Lambda_{0} \Rightarrow \ln I(\lambda) \geq(1-\delta) Q(\lambda), \\
I(\lambda) \geq \exp ((1-\delta) Q(\lambda)) .
\end{gathered}
$$


By (24) in Theorem 1, we have

$$
e^{C(\phi)} r[\zeta](\lambda) e^{\zeta^{*}(\lambda)} \geq \exp ((1-\delta) Q(\lambda))
$$

and

$$
\frac{\zeta^{*}(\lambda)}{Q(\lambda)} \geq \frac{C(\phi)+\ln r(\lambda)}{Q(\lambda)}+(1-\delta) \geq(1-2 \delta), \quad \Lambda(\lambda) \geq 2 \Lambda_{0}
$$

Therefore

$$
\zeta^{* *}(x) \leq \frac{1}{1-2 \delta} Q^{*}\left(\frac{x}{1-2 \delta}\right) .
$$

Applying the Fenchel-Morau Theorem, we conclude the proof.

To summarize.

Theorem 11. (Hybrid estimate). Suppose that all the conditions of Theorems 9 and 9 are satisfied. Then the following limit there exists and

$$
\lim _{\Lambda(x) \rightarrow \infty} \frac{\zeta(x)}{Q^{*}(x)}=1
$$

\section{An example.}

In this Section we consider $X=\mathbb{R}^{d}$ as well as $\lambda \in \mathbb{R}^{d}$.

Definition 3. Recall that the function $g=g(x): \mathbb{R}^{d} \rightarrow \mathbb{R}$ is said to be radial, or spherical invariant, iff it depends only on the Euclidean norm $|x|$ of the vector $x=\vec{x}$, namely there exists $g_{0}: \mathbb{R} \rightarrow \mathbb{R}$ such that

$$
g(x)=g_{0}(|x|)
$$

Lemma 3. Suppose that the function $g: \mathbb{R}^{d} \rightarrow \mathbb{R}$ is radial and such that its Young-Fenchel transformation $g^{*}(y)$ there exists. Then $g^{*}(y)$ is again a radial function, namely there is a function $g_{0}: \mathbb{R} \rightarrow \mathbb{R}$ for which

$$
g^{*}(y)=g_{0}^{*}(|y|)=\sup _{z \in \mathbb{R}}\left(|y| z-g_{0}(z)\right) .
$$

As a consequence, it is an even function.

Moreover, the optimal value in the definition of the Young-Fenchel transformation, i.e. the variable

$$
x(y)=x[g](y):=\operatorname{argmax}_{x \in \mathbb{R}^{d}}((x, y)-g(x)),
$$

so that $g^{*}(y)=(y, x[g](y)-g(x[g])(y))$, is also a radial function if, of course, there exists and it is uniquely determined.

PROOF. Let $U: \mathbb{R}^{d} \rightarrow \mathbb{R}^{d}$ be an arbitrary linear unitary operator and let $U^{*}$ be its conjugate (linear) operator, also unitary. Recall that a function $f: \mathbb{R}^{d} \rightarrow \mathbb{R}$ is radial iff for an arbitrary linear unitary operator $U$, it is $f(U x)=f(x), x \in \mathbb{R}^{d}$. 
We have

$$
\begin{aligned}
g^{*}(U y) & =\sup _{x \in \mathbb{R}^{d}}((x, U y)-g(x))=\sup _{x \in \mathbb{R}^{d}}\left(\left(U^{*} x, y\right)-g(x)\right) \\
& =\sup _{x \in \mathbb{R}^{d}}\left(\left(U^{*} x, y\right)-g\left(U^{*} x\right)\right)=\sup _{z \in \mathbb{R}^{d}}((z, y)-g(z))=g^{*}(y) .
\end{aligned}
$$

Therefore the function $g^{*}(y)$ is radial.

The second statment has an alike proof.

Remark 2. The radiality of the Fourier transform of a radial function is well-known, see e.g. [35, chapters 2,3].

Let us consider the following family of Young-Fenchel functions

$$
\zeta_{\kappa, L}(\lambda) \stackrel{\text { def }}{=} \begin{cases}\kappa^{-1}|\lambda|^{\kappa} L^{1 / \theta}\left(|\lambda|^{\kappa}\right), & |\lambda| \geq e \\ C \lambda^{2}, & |\lambda|<e\end{cases}
$$

where $\kappa=$ const $>1, \theta=\kappa /(\kappa-1), L(r), r \geq e$ is a slowly varying at infinity function, continuous and twice differentiable, such that

$$
\lim _{r \rightarrow \infty} \frac{L(r)}{L(r / L(r))}=1 .
$$

The Young-Fenchel transformation for these functions is calculated in particular in the monograph [34, chapter 1, sections $1,3,4]$ : as $x \rightarrow \infty$

$$
\zeta_{\kappa, L}^{*}(x) \sim \theta^{-1}|x|^{\theta} L^{1 / \theta}(x) .
$$

One can apply our theory of Tauberian theorems.

Theorem 12. Let $\zeta_{\kappa, L}(\lambda)$ be defined in (53). Denote

$$
I_{\kappa, L}(\lambda):=\int_{X} e^{(\lambda, x)-\zeta_{\kappa, L}^{*}(x)} d x
$$

We have

$$
\lim _{\Lambda(\lambda) \rightarrow \infty} \frac{\ln I_{\kappa, L}(\lambda)}{\zeta_{\kappa, L}(\lambda)}=1 .
$$

Furthermore, the inverse conclusion holds true. Namely, if for some Young-Orlicz function $\zeta=\zeta(x)$

$$
\lim _{\Lambda(\lambda) \rightarrow \infty} \frac{\ln I[\zeta](\lambda)(|\lambda|)}{\zeta_{\kappa, L}(|\lambda|)}=1
$$

then

$$
\lim _{\Lambda(x) \rightarrow \infty} \frac{\zeta(|x|)}{\zeta_{\kappa, L}^{*}(|x|)}=1 .
$$

A particular case:

$$
\zeta(x)=\zeta_{m, r}(x)=m^{-1}|x|^{m} \ln ^{r}(|x|), \quad|x| \geq e, m=\text { const }>1, r=\text { const } \in \mathbb{R} .
$$

We obtain, after some calculations, as $|y| \rightarrow \infty$, 


$$
\zeta_{m, r}^{*}(y) \sim \frac{1}{m^{\prime}}(m-1)^{r /(m-1)}|y|^{m^{\prime}}[\ln |y|]^{-r /(m-1)},
$$

where $m^{\prime}=m /(m-1)$.

\section{Concluding remarks.}

We have obtained bilateral asymptotic as well as non-asymptotic estimates for the multivariate Laplace integrals. Furthermore, we have presented multidimensional Tauberian theorems for exponential integrals.

A. It is interesting, by our opinion, to generalize the estimates obtained in Section 2 to the case of infinitedimensional linear spaces, as well as to generalize our estimates for the more general integrals of the form

$$
I[\zeta](\lambda):=\int_{X} \exp \zeta(\lambda, x) \mu(d x) .
$$

B. One can consider also the applications of the obtained results in the Probability theory, namely, in the theory of great deviation, asymptotical or not.

\section{Acknowledgement}

The first author has been partially supported by the Gruppo Nazionale per l'Analisi Matematica, la Probabilità e le loro Applicazioni (GNAMPA) of the Istituto Nazionale di Alta Matematica (INdAM) and by Università degli Studi di Napoli Parthenope through the project "sostegno alla Ricerca individuale".

\section{References}

1. D.R. Bagdasarov and E.I. Ostrovsky, Reversion of Chebyshev's Inequality, Probab. Theory Appl., vol. 40, no. 4, 737-742, 1996.

2. C. Bennet and R. Sharpley, Interpolation of operators, Academic Press, Inc., Boston, MA, 1988.

3. N. H. Bingham, Tauberian theorems and large deviations, Stochastics, 80, 143-149, 2008.

4. M. Broniatowski and A. Fuchs, Tauberian Theorems, Chernoff Inequality and the Tail Behavior of Finite Convolution of Distribution Function, Adv. Math., vol. 116, no. 1, 12-33, 1995.

5. V.V. Buldygin and Yu.V. Kozachenko, Metric Characterization of Random Variables and Random Processes, Translations of Mathematics Monograph, AMS, vol.188, 1998.

6. H. Chen, Evaluation of the Laplace integral, Internat. J. Math. Ed. Sci. Tech., vol. 35, no. 5, 773-777, 2004.

7. H. Chernoff, A career in statistics, In X. Lin, C. Genest, D.L. Banks, G. Molenberghs, D.W. Scott, J-L. Wang, Past, Present, and Future of Statistical Science. CRC Press. p. 35. ISBN 9781482204964, 2014.

8. H. Chernoff, A measure of asymptotic efficiency for tests of a hypothesis based on the sum of observations. Ann. Math. Statistics, vo. 23, 493-507, 1952.

9. P.L. Davies, Tail probabilities for positive random variables with entire characteristic functions of very regular growth, Z. Angew. Math. Mech., vol. 56, 334-336, 1976.

10. P. Eichelsbacher and L. Knichel, Fine asymptotics for models with Gamma type moments, ArXiv:1710.06484v1 [math.PR] 17 Oct 2017.

11. M.V. Fedoryuk, The saddle-point method, Moscow, Nauka (In Russian), 1977.

12. J.L. Geluk, L. de Haan and U. Stadtmüller, A Tauberian theorem of exponential type, Canad. J. Math. vol. 38, no. 3, 697-718, 1986.

13. J.L. Geluk, On the relation between the tail probability and the moments of a random variable, Nederl. Akad. Wetensch. Indag. Math., vol. 46, no. 4, 401-405, 1984.

14. S. Janson, Further examples with moments of Gamma type, arXiv:1204.5637v2, 6 Feb 2013.

15. S. Janson, Moments of Gamma type and the Brownian supremum process area, Probab. Surv., vol. 7, 1-52, 2010.

16. Y. Kasahara, Tauberian theorems of exponential type, J. Math. Kyoto Univ., vol. 18, no. 2, 209-219, 1978.

17. Y. Kasahara and N. Kosugi, Remarks on Tauberian theorem of exponential type and Fenchel-Legendre transform, Osaka J. Math., vol. 39, no. 3, 613-619, 2002

18. T.M. Lapinski, Multivariate Laplace approximation with estimated error and application to limit theorems, J. Approx. Theory, vol. 248, 105305, 2019. 
19. J. Korevaar, Tauberian theory: a century of developments, Grundlehren der Mathematischen Wissenschaften, vol. 329, SpringerVerlag, Berlin, 2004

20. N. Kosugi, Tauberian theorem of exponential type and its application to multiple convolution, J. Math. Kyoto Univ., vol. 39, no. 2, 331-346, 1999.

21. Yu.V. Kozachenko, Yu.Yu. Mlavets and N.V. Yurchenko, Weak convergence of random processes from spaces $F_{\Psi}(\Omega)$, Stat. Optim. Inf. Comput. vol. 6, no. 2, 266-277, 2018.

22. Yu.V. Kozachenko and E.I. Ostrovsky, The Banach Spaces of random Variables of subgaussian Type, Theory of Probab. and Math. Stat., (in Russian). Kiev, KSU, vol. 32, 43-57, 1985.

23. Yu.V. Kozachenko, E.I. Ostrovsky and L. Sirota, Relations between exponential tails, moments and moment generating functions for random variables and vectors ArXiv:1701.01901v1 [math.FA] 8 Jan 2017.

24. J.L. López and P.J. Pagola, An explicit formula for the coefficients of the saddle point method, Constr. Approx. vol. 33, no. 2, $145-162,2011$.

25. V.P. Maslov and M.V. Fedoryuk, Logarithmic Asymptotic behavior of the Laplace integrals, Mathematical Notes, vol. 30, no. 5, 763-768, 1981.

26. D.M. Mason, An extended version of the Erdös-Rényi strong law of large numbers, Ann. Probab., vol. 17, no. 1, 252-265, 1989.

27. G. Nemes, An explicit formula for the coefficients in Laplace's method, Constr. Approx., vol. 38, no. 3, 471-487, 2013.

28. G. Nemes, An Extension of Laplace's Method, Constr. Approx., doi.org/10.1007/s00365-018-9445-3, 2018.

29. E.I. Ostrovsky, Exponential estimations for Random Fields and its applications, (in Russian). Moscow-Obninsk, OINPE, 1999.

30. E. Ostrovsky and L. Sirota, Vector rearrangement invariant Banach spaces of random variables with exponential decreasing tails of distributions, ArXiv:1510.04182v1 [math.PR] 14 Oct 2015.

31. E. Ostrovsky and L. Sirota, Non-asymptotical sharp exponential estimates for maximum distribution of discontinuous random fields, ArXiv:1510.08945v1 [math.PR] 30 Oct 2015.

32. E.I. Ostrovsky, About supports of probability measures in separable Banach spaces, Soviet Math., Doklady (in Russian), vol. 255, no. 6, 836-838, 1980.

33. E. Ostrovsky and L. Sirota, Inversion of Tchebychev-Tchernov inequality, ArXiv:1711.06896v1 [math.PR] 18 Nov 2017.

34. E. Seneta, Regularly Varying Functions, Springer-Verlag, New York, 1976.

35. E. M. Stein and G. Weiss, Introduction to Fourier analysis on Euclidean spaces, Princeton University Press, Princeton, N.J., 1971.

36. A. Tauber, Ein Satz aus der Theorie der unendlichen Reihen, Monatsh. Math. Phys., vol. 8, no. 1, 273-277, 1987.

37. A.L. Yakimiv, Probabilistic applications of Tauberian theorems, Modern probability and statistics, VSP, Leiden, ISBN: 9067644374, 2005.

38. A. Zhang and Y. Zhou, A Non-asymptotic, Sharp, and User-friendly Reverse Chernoff-Cramer Bound. ArXiv:1810.09006v1 [math.PR] 21 Oct 2018. 